\title{
As long as you get paid: expectation of reward modulates executive inhibition
}

Paula M. Herrera ${ }^{1,2,3,4}$, Alberto Vélez Van Meerbeke ${ }^{3}$, Mario Speranza ${ }^{1}$, Claudia López Cabra

${ }^{3}$, Mauricio Bonilla ${ }^{5}$, Michaël Canu ${ }^{6}$ and Tristán A. Bekinschtein ${ }^{4}$

${ }^{1}$ HANDiReSP, Université de Versailles Saint Quentin en Yvelines, Le Chesnay, France

${ }^{2}$ Facultad de medicina, Universidad Tecnologica de Pereira, Colombia

${ }^{3}$ Grupo de investigación en neurociencias (NeURos), Universidad del Rosario, Bogotá,

Colombia

${ }^{4}$ Department of Psychology, University of Cambridge, United Kingdom.

${ }^{5}$ Laboratorio de Psicología Experimental, Facultad de Psicología, Universidad del Bosque, Bogotá, Colombia

${ }^{6}$ Departamento de Ingeniería Eléctrica. Universidad de Los Andes, Bogotá, Colombia

Corresponding author:

Paula M. Herrera ${ }^{1,2,3}$

Email Address: p.herrera@utp.edu.co 


\begin{abstract}
In order to address the question of the adaptive changes in executive inhibition induced by expectation, we designed a stop signal task where participants either knew or ignored the possibility of receiving distinct monetary feedbacks after each successful inhibition. Our results confirmed previous observations demonstrating a "kick start effect": that the presence of a high reward feedback at the beginning of the task has the potential to enhance response inhibition, compared to when participants start with low reward, getting high reward at the end. The progression of inhibition scores (SSRT) evidenced a progressive delta change between the 1 st and the following trial blocks when participants were aware of the reward shift. In contrast, the unexpected reward shift group exhibited a main change between the no rewarded and the first rewarded block, with no further changes on the SSRT scores. Our findings suggest that the cognizance of reward magnitudes seems to play a crucial role in executive modulation of inhibition.
\end{abstract}

Key words: motivation, reward, expectation, executive inhibition, kick start effect

\title{
Introduction
}

The concept of inhibition in human cognition can be approached from its basic motor and reflex aspects, till more elaborated inhibitory processes as the planned action under particular circumstances [1]. Inhibition can also be defined as the resistance to interference [2]. Seen from a cognitive perspective, inhibitory control is not only a fundamental tool to guide behaviour towards accomplishment of goals. It also implies the ability to dynamically modify or cancel planned actions [3]. Such cognitive inhibition is more long-lasting in time, compared to neural inhibition. This dynamical dimension of inhibition is crucial to enable the flexibility of cognitive and behavioural control systems [4].

The stop signal task (SST) has been widely studied in recent years [5]. It is considered that the tested process is reactive control, which is the way how subjects stop a response straightforward when instructed by a signal [1]. A good and well known example of this process is the classical version of the GoNoGo task [6]. This kind of task depends on 
immediate stimulus. Although, some authors consider that the tested process during the Stop signal task is more related to the withholding capacity, giving the features of the task: a motor response is demanded at every trial, unless a stop signal appears after the Go signal. This has been designed as proactive inhibition: the ability to prepare to stop due to a possible upcoming signal. Such proactive inhibition is supposed to be controlled by subject's internal states, such as goals and motivation [38]. Beyond the reactive and proactive motor control, there is the behavioural stopping, implying more complex hierarchy in a control system relying not only on the motor inhibition ability but also on attention, memory, and, inner motivational states, closely related to the reward system [7]. Defining distinct kinds of inhibition is still the core of theoretical debates [8,3]. Despite these limits, it is widely accepted that the Stop signal task is a suitable model to explore cognitive inhibition [9].

Mirroring those somewhat fuzzy boundaries when defining inhibition, the finest underpinnings of its neural mechanisms have led to some remarkable discoveries. Based on current knowledge, it seems possible to induce some adjustments to the inhibition profile. Experiments applying subtle manipulations to the Stop Signal Task have been successful in showing distinct within-subject effects. One example is the modulation of the cost of failures of inhibition. If a punishment is introduced after a failed inhibition, the participant gets better scores on the stop signal reaction time (SSRT) and the number of failed stops drops [10]. This inhibition improvement is also possible if successful inhibition is rewarded $[11,12,13]$. Also a learning effect may be induced by training through similar presentation patterns of the stop signal [14]. There has been an increasing interest in the study of the role of reward over the modulation of inhibition, but few experiments have directly explored the effect of distinct reward values in humans $[15,16]$

In the present work, we offer more experimental evidence to confirm that the modulation of inhibition can be affected by different reward magnitudes [17]. Moreover, beyond reward magnitudes itself, the reward expectation seems to play a crucial role on the behavioural adjustment through an inhibition task. This effect has been described by Brown and Braver [18], and Jessup et al [19]. Shenoy and Logan have proposed additional insights to the Horse Race model $[20,21]$ to explain some aspects of the dynamical adjustments through the Stop Task, when motivational parameters are manipulated. The so-called rational decision-making 
framework highlights the role of the sensory process and the action choice depending on the cost of inhibition errors [22].

In a previous study on a Stop Signal task modified by reward levels, [17] authors have suggested two effects of the monetary reward modulation over inhibition: an effect of the reward size itself and a kick start effect, suggested by the up-modulation of the highest reward at the beginning of the task. Subjects were aware of the reward magnitude assignment before performing the task. However, one can wonder about what amount of bias could be induced in behavioural adjustments by advance knowledge of reward. In other words, what is the role of expectation of reward on inhibition performance.

Our next step has been to ask: What happens when subjects know they can win a high or a low reward, but they are not aware of the order of reward assignments? And what happens if subjects know about the presence of a monetary reward through the inhibition task, but they are not told about the presence of different reward magnitudes?

One suitable hypothesis could be based on the predictive error minimization [21]. In order to find the optimal strategy, the subject accumulates sensory evidence that allows the identification of the minimal error probability and decision delay, leading to a decision threshold. Getting a reward during successful inhibitions can enhance the withholding strategies during a stop task. Then, it is tempting to push further the question about the reward magnitudes over the predictive adjustment of behaviour.

In the aim to answer to these questions, we have conducted a Stop Task Paradigm with four blocks over two different groups. One group was aware of the possibility of rewards magnitudes shift but the order of rewards was not communicated. In the other group, subjects only knew that a monetary reward will appear without any mention to the reward shift and they discovered a distinct reward magnitude only at the last block.

In the "expected reward shift" group, we awaited the same results founded on our last paper: kick start effect when subjects received a high reward at the beginning, and a modest effect of the reward magnitude itself. 
In the "unexpected reward shift" group, we predicted to find a kick-start effect over all subjects at the first monetary rewarded block independently of the size of rewards, and an extra boost effect on performance over the blocks offering the highest reward.

\section{Methods}

\section{Participants}

Young adult participants were recruited by informal community announcements among undergraduate students attending at the Universidad El Bosque and at the Universidad del Rosario in Bogota (Colombia). Both universities granted Ethical approval to carry out the study within its facilities by formal letter in response to written demand requested by the research team, and following the internal procedures of each university.

Forty-five participants were recruited from both universities. A random assignment allowed the constitution of two main groups. Twenty-one participants were assigned to the "Expected reward shift" group, and twenty-four to the "Unexpected reward shift" group. The combined mean age for both men and women participating from the study was 22.6. (Age range 20 - 31, $\mathrm{sd}=4.5)$. Sex ratio in the "Expected reward shift" group was 1.2, and in the "Unexpected reward shift" group of 1.1 .

\section{Procedures}

Participants were screened for past and current psychiatric disorders. An open questionnaire was conducted in the search of history for Autism Spectrum Disorders, Learning Disorders, Attention Deficit/Hyperactive Disorder (ADHD), mood disorders (depression or bipolar disorders) and schizophrenia, as these were part of the exclusion criteria. All participants gave written consent according to the procedures of the Ethical committee of the Universidad del Rosario and Universidad El Bosque in Bogota, Colombia.

All time responses (mean reaction time [MRT], stop signal delay [SSD] and stop signal reaction time [SSRT]) were screened for outliers, given a cutting point of $+/-2$ standard deviations from the mean response value. Two participants were excluded from the study after applying these criteria. 
Experiment environment conditions were controlled. All participants performed the experiment in a specific room arranged by each laboratory of experimental psychology at each University (del Rosario and El Bosque). Each room was equipped in a similar way, containing a desk in a fixed position, a computer and the EEG acquisition machine. Participants filled out written information about the study and a consent form in paper form before performing the task.

In order to assess behavioral inhibition, participants performed our Stop Signal Task paradigm, modified by monetary reward levels. The general principle of the Stop Task is a routine motor reaction where participants must hit a key each time he or she is confronted with a frequent go stimulus, and a cancellation of the ongoing action, after exposure to an infrequent stop signal appearing after the go stimulus.

Our visual stimuli and experimental design consists on a modified version of the Stop Signal Task developed by Rubia and colleagues (2003) [23], which is, in turn, a faster visual variant of the Tracking Stop Signal Task [20]. Main modifications reside on the introduction of monetary feedback after each successful inhibition, and the suppression of punishment feedback after a failed inhibition.

Three type of feedback were granted: a no monetary feedback given by a Smiley, and two types of monetary rewards: low reward by fifty Colombian pesos (50\$ COP) and high reward with five hundred Colombian pesos (500\$ COP).

Each block was assigned to with a specific feedback to build up two conditions, given the order of presentation of monetary feedbacks (See Figure 1). Both conditions started by a no monetary reward on the first block (Smiley). Increasing conditions conferred a low reward on the 2nd and the 3th blocks (50\$ COP) and a high reward at the end (500\$ COP on 4th block). Decreasing conditions granted the high reward (500\$ COP) on blocks 2 and 3, and the low reward (50\$ COP) at the end.

Participants were randomly assigned to essays conditions in a counterbalanced way. Half of participants underwent the Increasing Condition and the other half, the Decreasing Condition. This experimental arrangement was set to avoid bias given by the order of presentation of rewards. The order of presentation of rewards was unknown to all participants. 
The key point of our present experimental design was the fact that some participants were expecting different monetary rewards magnitudes ("expected reward shift" group), and the others only knew that a monetary reward will appear ("unexpected reward shift" group).

Figure 1. Experimental design. Stop signal task with reward magnitudes

\section{[INSERT FIGURE 1 HERE]}

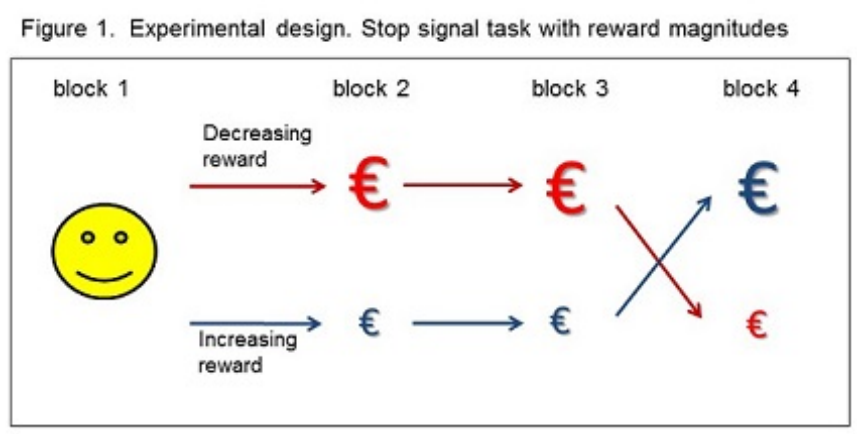

[Legend] Stop signal task with reward magnitudes. All participants underwent the same task, starting with no monetary feedback, then split on increasing or decreasing monetary reward conditions.

Instructions for the Stop Signal Task were presented in a standardized paper form and delivered by the same experimenter. Participants were instructed about a video game-like task to determine how fast they were. They were told about the length of the task, comprising 4 blocks, with a short pause between blocks. After giving the instructions, participants were asked to repeat the procedure to the experimenter in order to verify the full understanding of the task. When needed, questions were answered. A brief training block of the Stop Signal Task without feedback for successful inhibition was undertaken before beginning the trials.

\section{Measures}

Statistical analyses were performed with SPSS (IBM SPSS Software Statistics Developer 21.0.0.0 Version, 2012) and $\mathrm{R}$ (R version 2.13.0 (2011-04-13) Copyright (c) 2011. The $\mathrm{R}$ Foundation for Statistical Computing). All data were checked for outliers, normal distribution and homogeneity of variance. Critical alpha was set at .05 but frequently adjusted using Bonferroni corrections. 
As usual for the Stop Signal Task analysis, dependent variables consist on three type of response time measures (MRT, SSD, and SSRT), and four task performance measures (number of failed inhibitions, missed go's, wrong keys and number of rewards).

Stop signal reaction time (SSRT) was generated through the mathematical model proposed by Logan and collaborators (Logan et al., 1997), following a subtraction of the Mean reaction time (MRT) minus the Stop signal delay (SSD) (formulae SSRT = MRT - SSD) [24].

Multiple analyses levels were conducted, given the complexity of the data.

In the first step, each group was analyzed separately (Expected / Unexpected reward shift), and a final step applying common analyses to the whole group.

The first step started by individualized analyses of each reward order condition (Increasing reward/ decreasing reward). Every time performance variable (MRT, SSD, SSRT) and task performance measures was analyzed through paired samples T-tests. Secondly, One Way ANOVAs were conducted for each independent group (Increasing and Decreasing Reward) to explore the modulation effect of the reward magnitudes per group. Then, a Univariate General Linear Model (GLM) was applied with the aim to analyze similar time blocks between increasing and decreasing reward conditions, comparing first block on increasing reward with the correspondent first block on decreasing reward, then second blocks and so on. Afterwards, the whole trial group was analyzed through Two -Way ANOVAS as follows: within-subject factors 'order of blocks' (1,2,3... given by the acquisition block order), 'type of reward' (smiley, low reward, high reward) and between-subject factor 'condition' (Increasing reward, decreasing reward). Post Hoc analyses were conducted through Bonferroni tests.

Finally, a delta change analysis was conducted with the aim to search dynamic progression differences through the task over inhibition scores.

\section{Materials}

The task was programmed in Visual Basic 6.0. DELL personal computer with an Intell 2 processor was employed to run the task. Participants were placed in a desk chair without wheels, at a standard distance of $1 \mathrm{~m}$ from a 20 " screen. Stimuli appeared against a black background at the center of the screen. Alignment of the head was coordinated through visual verification, between the participant nose and the fixation cross at the center of the screen. 
This alignment permitted to ensure that stimuli would be displayed in the middle of the visual field.

The testing room was artificially lighted, with no visual distractors on the walls and without windows, in order to avoid all attention-grabbers. At the beginning of the task, the participants underwent a short practice block, ensuring the correct visualization of every stimulus; luminosity was kept constant on the stimuli with no ambiguity.

\section{Availability of data and materials}

The dataset supporting the conclusions of this article is available in the University of Cambridge data repository in http://www.data.cam.ac.uk/repository [DOI will be informed]

\section{Results}

\section{Stop signal task under expected reward shift condition}

Twenty-two participants between 20 and 25 years old performed the Stop Task on four blocks. Normality was checked using Shapiro-Wilk test. Analysis of normality exhibited a normal distribution allowing parametric analyses. One subject was excluded due to laterality errors, which resulted in making precision errors beyond 2 SD away from the group mean score. Analyses were applied over a group of 21 participants.

Instruction consisted of the classical Stop Signal Task, described on the methods section. Additional information was given concerning the presence of distinct reward magnitudes, omitting any precision of the order of reward assignment. Participants were blind to the existence of increasing or decreasing conditions.

Statistical analyses were performed on two levels. A first level was conducted over each independent condition (increasing / decreasing reward) to evaluate performances on time, and a second level comparing performances related to reward sizes. Full behavioural data and figures are presented in Additional Table 1 and Figure 1.

\section{Performances through time}


Paired sample T-tests were conducted to compare differences between each block given their presentation timeline inside each condition (blocks (b) comparison as follows: b1-b2; b1-b3, b1-b4, b2-b3, b3-4). Correction for multiple comparisons was not applied in this step. Alpha level was set at .05. Main differences were observed between each block comparison inside each condition, except between the 2 nd and the 3th blocks. This is an expected outcome, given the fact that feedback was the same on blocks 2 and 3. No difference was found for MRT on decreasing neither on SSRT for increasing condition (see Additional Figure 1 and Table 1 online for full results).

Failed stops on increasing condition did not show any difference among each reward shift. On decreasing condition, a difference was seen between block 1 and each subsequent block.

No difference was obtained on missed go's comparing blocks. Wrong keys presented paired differences on increasing condition between block 1 and all other subsequent blocks. Regarding rewards, paired differences were present inside the increasing condition for comparison between block 1 and 4, block 3 and 4, and a trend between block 2 and 4 (see Additional Figure 1 and Table 1 online for full results).

\section{Performances and reward size}

Paired samples T-test comparing each reward magnitude (smiley Vs low reward, smiley Vs high reward, low reward Vs high reward), revealed an effect of reward magnitude between the non-monetary reward (smiley) and the high rewards on increasing condition for MRT $(p=.034)$ and $\operatorname{SSD}(p=.01)$. No difference was observed between smiley and the first low reward on SSRT scores. For Decreasing condition an effect was observed on SSD between smiley and the first high reward $(p=.015)$, smiley Vs $2^{\text {nd }}$ high reward $(p=.004)$ and SSRT scores between smiley Vs $1^{\text {st }}$ high reward $(p=.009)$, and $2^{\text {nd }}$ high reward $(p=.004)$.

For task performance measures, paired simple t-test exhibited no difference on increasing condition except for wrong keys (smiley Vs $1^{\text {st }}$ low $(\mathrm{p}=.028)$ and Vs high reward $\left.(\mathrm{p}=.007)\right)$. Main differences on decreasing condition for Failed stops were observed between smiley Vs $2^{\text {nd }}$ high reward $(\mathrm{p}=.031)$. No differences for missed go's nor Wrong keys. Rewards exhibited a main difference between smiley Vs high reward on increasing condition $(\mathrm{p}=.018)$. 
Variances measured through SD values have shown a progression on each order condition (increasing/decreasing reward). Consistently, SD scores were higher on increasing condition, except for the inhibition measures (SSRT). A main difference was obtained through Univariate GLM on SSRT scores $(\mathrm{F}(1.19)=4.81, \mathrm{p}=.004)$ on 3 th and $4^{\text {th }}$ blocks $)$.

\section{Comparisons between increasing and decreasing conditions}

One-Way ANOVA comparing reward magnitudes on both conditions was performed, specifically for the search of the effect induced by the diverse reward magnitudes (no monetary reward (smiley), low reward and high reward, and the influence of the reward order assignment. Alpha level was set at .05 . A significant effect was seen between smiley and high reward blocks for the SSD measure $(\mathrm{F}(2,81)=3.49, p=.035)$ (See figure 2A, for "Expected reward shift" group). No difference for any task performance measure was obtained.

A Univariate General Linear Model (GLM) allowed comparisons among the two condition (increasing and decreasing rewards) through time, by correlations between similar time blocks as presented chronologically (first block from increasing condition Vs first block from decreasing condition, and so on). No main effect, but a trend was detected on SSRT scores among the third blocks on each condition $(\mathrm{F}(1,19)=3.42 ; p=.08)$,

Two Way-mixed ANOVAs were applied to explore the effect of the manipulation of reward orders given by the two reward order conditions (increasing or decreasing rewards), through the four blocks of the task (a $2 * 4$ mixed model). Alpha level was set at .025 . Main effect of group was found for all measured variables $(\mathrm{MRT}(\mathrm{F}(3,57)=5.16, p=.003), \operatorname{SSD}(\mathrm{F}=13.1$, $p<.001), \operatorname{SSRT}(\mathrm{F}=3.87, p=.002)$, but no effect of Order nor Group*Order interaction (For all results see Additional Information Table 2).

For task performance measures, a main effect of group was obtained for failed stops, wrong keys and number of rewards. An effect of order was seen for failed stops and rewards, and a group*order interaction effect was obtained for number of wrong keys.

Delta ratio slopes were obtained through the comparison of percent change between the first block and each following block. This mathematical strategy permitted a correlation between the starting point at the first block (no monetary reward) and the influence of the monetary 
rewards introduction over the trial (see figure 2B, for "Expected reward shift" group). A Univariate GLM highlighted significant differences over SSRT delta scores between the 1st and the last two blocks (block1 Vs block $3=\mathrm{F}(1,19)=4.97, p=.038$, block 1 Vs block $4=$ $p=.034)$. This steeper slope is clear on figure $2 \mathrm{~B}$ for "Expected reward shift" group decreasing condition on red).

Figure 2. Time performances for Expected and Unexpected reward shift groups

[INSERT FIGURE 2 HERE] 
Expected rewards

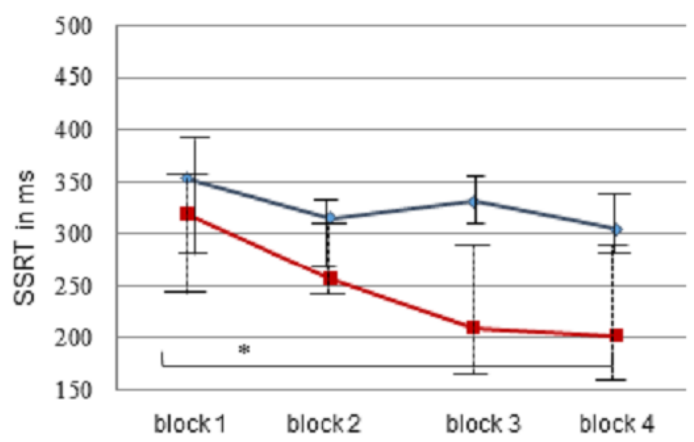

Increasing condition
Unexpected rewards

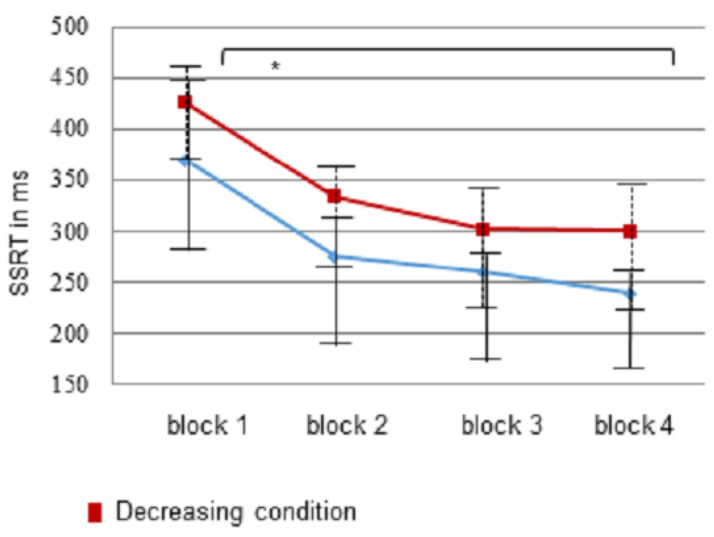

B) $\triangle$ SSRT

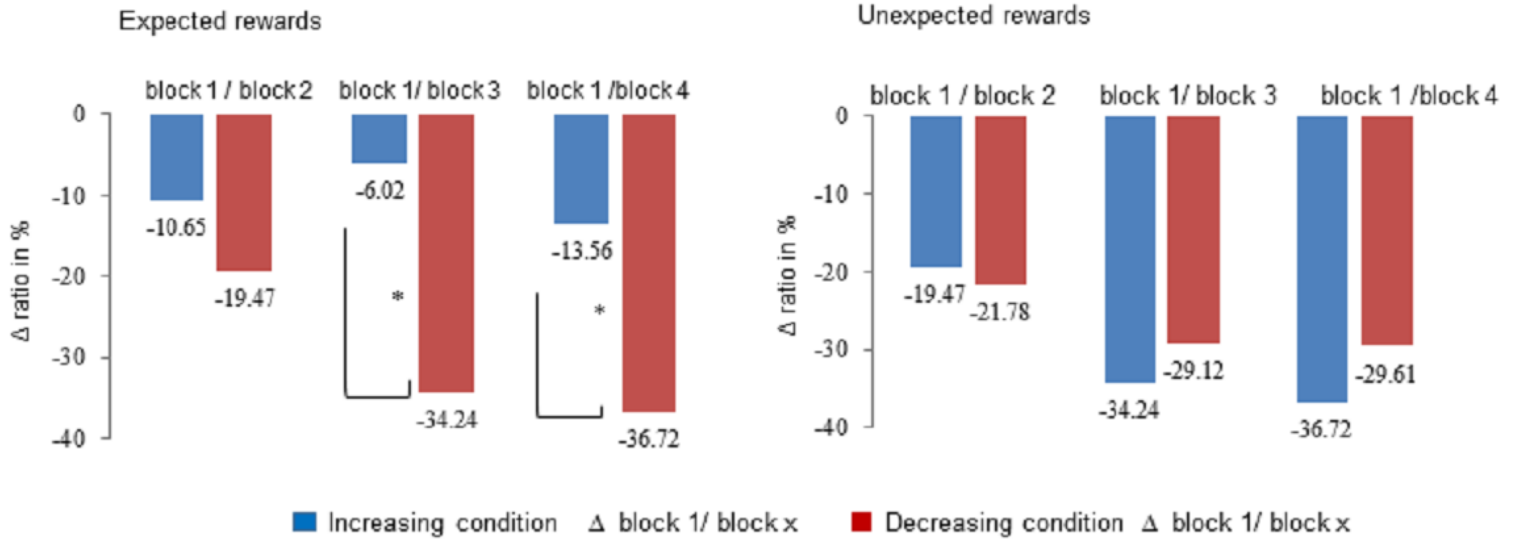

C) $\Delta$ SSRT No monetary Vs Monetary Rewarded blocks

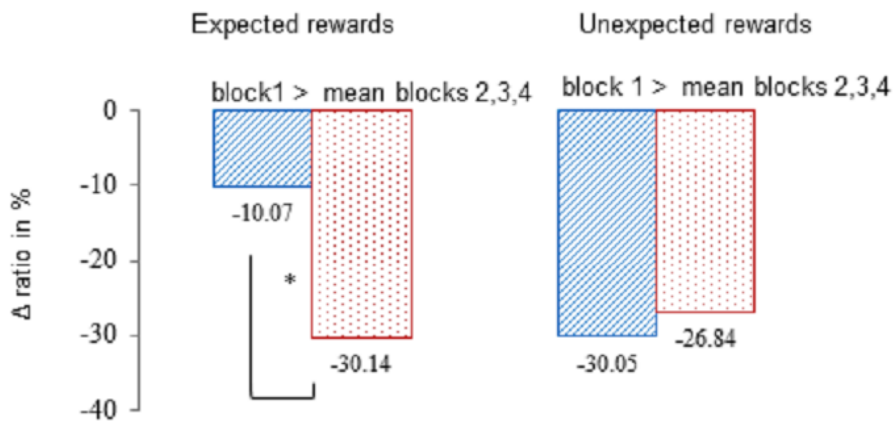

[Legend] 2A. SSRT= stop signal reaction time, increasing condition on blue, decreasing condition on red. Left: (Expected shift group) Main differences were obtained on paired comparisons by t-test between the first no rewarded and the following rewarded blocks but only on the decreasing condition (block 1/block2=p=.025, block 1/block3 $p=.004$, block1/block4= p=.009). Right (Unexpected shift group): main differences on both conditions were described between the first no rewarded and the 
following rewarded blocks condition (block $1 /$ block $2=p=.004$, block $1 /$ block $3 p=.005$, block $1 /$ block $4=p=.003$ )

2B. SSRT delta change ratio in percent between first block without monetary reward and 2nd, 3th and 4ths blocks with monetary reward. 2B Left: delta changes inside increasing and decreasing reward conditions on "Expected of reward" group. * Significant difference between delta slopes comparing increasing and decreasing condition for 3 th $\left((p=.038)\right.$ and $4^{\text {th }}$ blocks $(p=.034)$. 2B Right: delta changes inside increasing and decreasing reward conditions on "Unexpected of reward" group.

2C. SSRT delta change ratio in percent between Expected and Unexpected group, between Increasing and Decreasing reward conditions. *Difference was found between conditions on the "Expected reward shift" group. Main difference was found on the delta change between the no monetary and the monetary rewarded blocks $(\mathrm{F}(1.20)=4.14,(p=.05)$.

\section{Stop signal task under unexpected reward shift condition}

Twenty-four healthy adults underwent the SST over 4 blocks. After normality checking with Shapiro-Wilk Test and Outliers inspection, one subject was excluded due to slow responses falling outside 2 SD from the group mean on time performance measurements.

Participants were instructed about the Stop Signal Task. They were told about the length of the task (four blocks of 4 minutes each) and the presence of monetary reward, without mentioning reward magnitudes differences.

The same statistical analyses as for the Expected reward shift group were performed, as described in the methods section.

\section{Performances through time}

Paired sample T-tests were conducted to compare differences between each block inside each condition group. Alpha level was set at .05. Main differences were observed over the transitions between the first and all the following blocks, for MRT, SSD and SSRT values. No differences were reported in paired comparisons between $2^{\text {nd }}$, 3th and $4^{\text {th }}$ blocks, except for the SSD values in the decreasing condition. (see Figure 2 and Table 3 for data report on Additional Information).

In regard to task performance measures, paired sample t-tests on failed stops exhibited significant differences on increasing condition, except between block 2 and 3. On decreasing condition, differences were seen between b1-b4, b2-b4, b3-b4. No differences for missed Go's 
but a trend was observed between block 1 and 4 on increasing condition. For the number of wrong keys, paired differences were observed on decreasing condition between block 1 and 4 . On increasing condition a paired difference was observed between block 1 and 2. Concerning number of rewards, there was a main difference among all paired tests except between the last two blocks (b3-b4) on increasing condition. For decreasing condition, paired differences were observed between all comparisons except between the first two blocks ( b1- b2).

\section{Performances and reward size}

Paired samples T-test comparison for reward magnitudes inside each condition group highlighted a main effect on the transition between no monetary reward (smiley) and the first monetary reward. Moreover, inhibition scores given by the SSRT values reflect a robust effect of the presence of reward independently of being high or low and independent of its arrival order (SSRT paired T-test between smiley and the first monetary reward $=p=, 004$, smiley and the second monetary reward $=p=.005$, smiley and the last monetary reward $=p=.003$ ).

For task performance measures, all paired differences were significant for failed inhibitions, wrong keys and rewards, between Smiley Vs the first monetary rewards on block 2 and 3, independently of the reward value. No difference was seen between the third and the last block, regardless the reward size. Missed go's exhibited no difference between smiley and low reward on increasing condition.

\section{Comparisons between increasing and decreasing conditions}

One Way ANOVA $(\alpha<.05)$ was carried out to explore the effect of reward magnitudes through comparison of blocks with non-monetary feedback (Smiley), low and high reward. A main effect of reward was perceived on all time performance measures $(\mathrm{MRT}(\mathrm{F}(1,22)=3.32$, $p=.04), \operatorname{SSD}(\mathrm{F}=19.11, p<.001), \operatorname{SSRT}(\mathrm{F}=5.52, p=.005)$. Pairwise comparisons highlighted the strongest interaction between smiley and high reward. No significant differences were obtained between low and high monetary rewards.

A Univariate General Linear Model (GLM) was conducted in order to compare performances between the two conditions (increasing and decreasing reward) through equivalent blocks (block 1 of increasing condition against block 1 of decreasing condition and so on). Only the 
MRT scores were significantly different between the first blocks on above conditions $(\mathrm{F}(1,22)$ $=4.31, p=.05$ ). No differences were observed for any task performance measure.

Two-Way mixed ANOVAs $(\alpha<.025)$ were performed to explore the effect of the manipulation of reward orders distributed on two groups (increasing or decreasing rewards), through the four blocks of the task (a $2 * 4$ mixed model).

A robust effect of group was observed in all time measures $(\mathrm{MRT}=\mathrm{F}(3,66)=10.41 ; p<.001$, $\mathrm{SSD}=\mathrm{F}=23.61 ; p<.001), \mathrm{SSRT}=\mathrm{F}=8.105 ; p<.001)$, and failed stops $(\mathrm{F}=17,5(p<0,001))$, wrong keys $(\mathrm{F}=3.98, p=.05)$, and Rewards $(\mathrm{F}=22,18, p<0,001)$. (see Additional Table 4 for more detailed results). There was no effect of order over performances, produced by the reward order of presentation. Furthermore, there was no effect of interaction between the variables and the order of presentation of rewards ("increasing or decreasing condition" independent factor).

Finally, delta slopes analysis was made through comparisons between percentages of change of the first block and each following block, between both conditions (increasing and decreasing reward). A significant difference was observed between smiley and all the monetary rewarded blocks. However, no difference was seen between the increasing or decreasing conditions. The whole group had a similar progression pattern, which is a strong delta slope between the smiley and each monetary reward level, but no differences with the Univariate GLM analysis comparing blocks with monetary feedback (see Figure 2B "Unexpected reward shift" groups).

\section{Comparative outcomes between expected and unexpected reward shift groups}

First, a group analysis for blocks comparison between trials (Expected Vs Unexpected Reward shift) and between conditions (Increasing Vs Decreasing) through a Univariate GLM, was analysed, allowing the comparison of each time-corresponding block through both trials (blocks 1 on Expected and Unexpected trials, blocks 2 on both trials, and so on). MRT and SSD scores exhibited significant group differences over all paired block comparisons between 
trials (Expected Vs Unexpected). Only a main effect of order (increasing Vs decreasing reward) was seen on MRT among "blocks 1" comparison $(\mathrm{F}(1,37)=5,36, p=0,02)$.

A second Univariate GLM was conducted for trials and conditions comparisons, given each feedback value (smiley, low reward (50\$ COP), high reward (500\$ COP). A main effect of group was highlighted for each reward magnitude, under the Expected Vs Unexpected reward shift trials. No main effect of order of assignment of reward was seen, related to the increasing or decreasing reward condition.

A general crossed comparison was applied through a Two-Way (Two Factor) repeated measures for performances scores. A main effect of group was obtained on all time performance measures (MRT $(\mathrm{F}=16,45, \mathrm{p}=0,001), \operatorname{SSD}(\mathrm{F}=39,38, \mathrm{p}=<0.001), \operatorname{SSRT}(\mathrm{F}=13,71$, $\mathrm{p}=<0.001)$.

A main effect of the Trial (Expected Vs Unexpected) was seen on MRT $(F(1,37)=7,69$, $p=0,012)$ and a trend was seen over SSD scores $(\mathrm{F}(1,37)=5,57, p=0,03))$. Bonferroni post Hoc comparisons showed that main differences come from each first block and the following blocks, which highlights what was described earlier for each trial analysis: there is an important step on behaviour inhibition modulation between a non-monetary reward and the first monetary rewarded block. Moreover, delta changes analyses exhibited a main difference between the first block and the following ones, however only for the Expected reward trial $(\mathrm{F}=4,14,(p=0,05)$. This can afford the hypothesis about the role of being aware of reward magnitudes in the adjustment of motivational driven behaviour.

The presentation of the $\$ 500$ coin could have induced a stronger stability on the prediction system, reflected on significant shorter SD on MRT scores after the first block (2nd block's contrast on increasing vs decreasing conditions obtained on Univariate GLM for SD values = $p=, 01$, for 3th blocks: $p=, 001,4$ th blocks: $p<0,001$ ) (for full data see additional table $\mathrm{x}$ ).

\section{Discussion}


In the present study, we have conducted a stop signal task under two controlled conditions. First, we have introduced different reward magnitudes with the aim of manipulate the motivational dimension of the inhibitory task and see in which extent the reward size matters. Second, we have tested the effect of the previous knowledge of reward over the behavioural adjustments (Expected and Unexpected reward shift trials).

\section{"Expected reward shift" experiment}

Results showed a main effect for each group on all variables, enabling us to confirm that experimental manipulation induced a modulation effect over behaviour. Two main observations are made: there was an effect on inhibition performances induced by the order of reward presentation, and an influence of the monetary reward magnitude.

The effect in the order of reward assignment was observed through differences between increasing and decreasing conditions. The increasing reward condition group exhibited a discrete change on the performance pattern, with a surprising less efficient inhibition score on the third block, and the expected although modest improvement at the end, when receiving the high reward.

Concerning the decreasing condition group, significant differences on paired sample T-tests on number of failed stops and SSRT scores were observed between the first block that gives non-monetary feedback (smiley), compared to the subsequent monetary rewarded blocks. The dynamical progression of SSRT performance through the task exhibited a stronger delta change between the 1 st and the 3 th and 4 th blocks on the decreasing condition group.

Given the presence of the high reward in the $2^{\text {nd }}$ block, it was expected to also have a significant delta change at this point but this was not the case. Instead, it was observed a dynamical improvement progression of performance among the four blocks. Participants exhibited a better immediate global inhibition strategy under decreasing conditions in comparison with the increasing conditions. The presence of the strongest reward at the beginning of the monetary rewards assignment seems to play a crucial role over inhibition behaviour when subjects are aware of the reward amounts. This initial boosting effect looks more substantial than the promise of a high reward later on the task. A similar kick start effect has been described in a previous report on a Stop Signal Task [17], in which participants were told in advance about the exact moment when they were going to get a high or low reward. In contrast with the present work, inhibition performances mirrored the size of rewards: low 
reward, bad scores, higher reward, better performance. No such linear effect has resulted in the present work.

By manipulating the cognisance of the reward type, we wanted to go beyond the kick star effect and explore the effect of expectations over executive inhibition. If we stick to the assumption that merely the reward size would be the cause of a performance boost, we could expect a simple replication of previous work. Although, current results in both conditions exhibit a non-linear progression that does not strictly follow the reward size modulation.

We can claim that expectations about rewards to come modifies the way participants have adjusted the inhibitory strategies.

Given the manipulation of information about reward shift, we have certainly induced an expectation that works through the ongoing task course. When subjects "discover" the size of the reward at the second block, they can predict what is coming next, would it be another high reward or a low one. At the third block, the prediction becomes a certainty: if you get another high reward there is no doubt that the reward size shift will come in the fourth block.

In regard to the increasing conditions group, we may have induced an undermining effect. The presence of a low reward, when you are expecting a high one, may have acted as a demotivating or non-attractive reward. This demotivation is perceived through a nonsignificant inhibition improvement when compared to the group receiving the high reward in that moment of the task. The undermining effect can be confirmed at the third block, when subjects are confronted again with a low reward, showing worse inhibition scores than the previous block. Finally, when these participants received the highest reward, inhibition scores come to improve and were the best among all trials, with a significant difference between the first and the last block on number of obtained rewards.

"Unexpected reward shift" experiment

Analyses showed a main effect of group on all time performance variables (MRT, SSD, SSRT), and over failed stops, number of rewards and number of wrong keys, which confirms the modulation effect on behaviour induced by the experimental manipulation.

Following the same procedure as for the "Expected reward shift" group, participants did not realize that they belonged to one of the two existent conditions (increasing and decreasing 
reward). Additionally, we have suppressed the information about the existence of distinct reward magnitudes.

As expected, results showed a progressive improvement on all measured scores, independently of the condition, and regardless to the reward magnitude order. This supports a general kick start effect on performance, after the introduction of a monetary reward on the task.

Slopes between blocks and between the two conditions were quite similar for all measures. The steeper slope was observed between the first and the second block. This corresponds to the shift between a non-monetary feedback (smiley) and the first monetary reward, no matter if it concerned a low or a high reward. These findings comfort the hypothesis that on unexpected reward shift trials, the modulation effect of monetary reward would be induced by the presence of the reward itself, independently of its magnitude. Moreover, the lack of information about rewards shift, restrains a possible dynamical modulation of expectation through the task. The hypothesis about the reward magnitude effect was not confirmed. An extra boost of high reward at the end, or an undermining effect of the low reward was not observed. Instead, we can claim the up modulation of the inhibitory system by the mere presence of any amount of monetary reward. Instructions given to the "unexpected reward shift" group may have induced a single boot expectation that works pretty well in the inner motivation, placing any amount of money at the top of expected feedbacks. The kick start effect may have operated in a similar way than the one induced by the presence of explicit high reward at the beginning of the task.

\section{Conclusions}

Three main components of inhibition can be tested with the Stop Signal Task modified by reward levels: the motor/proactive inhibition, the cognitive inhibition and the influence of motivation. The cognitive aspect is given by an overt instruction to restrain the action under a very specific circumstance, leading to several requirements: first the understanding of the rule given by verbal instruction and then requiring the retention of information in working memory. The following withholding strategy seems enhanced by motivation, in addition to the pro-active/planned inhibition capacity [8]. The motivational aspect is driven by 
modulations of performances in front of distinct types of rewards, would they be monetary or not.

Here we claim that expectation may constitute an extra element to take into account when testing the motivational effect of reward over executive inhibition, inducing an influence on both the cognitive dimension as well as on the inner motivation.

In the present work, we have addressed the question about the effect of expectation on a rewarded inhibitory task by asking if there is a difference on performance when participants know they can win a high or a low reward, compared to participants that do not know about reward differences. The answer is yes. From one side, a robust effect was obtained through analyses comparing increasing and decreasing conditions inside the "Expected reward shift" group. Participant receiving the highest reward at the beginning of the task have modulated the inhibition pattern in a more efficient way than those starting with low reward. Moreover, the dynamical progression of SSRT scores on the decreasing condition was similar to previous study reporting the kick start effect. Even if the order of the reward shift was unknown, participants received the higher reward at the beginning and were able to deduce what amount of money could come next. From the other hand, subjects not knowing about the presence of distinct reward magnitudes have reached a ceiling pattern right after receiving the first reward, would it be high or low. This observation suggests that participants only had a single expectancy: receiving monetary reward. Moreover. the behavioural adjustments in front of unexpected reward shift were similar to the one exhibited by the subjects obtaining the kick start effect. Allegedly, the best effort was disposed promptly at the bonus arrival moment.

“As long as you get paid" seems good enough for modulating inhibition, as stated by some studies $[15,16,40]$. The novelty of the present work is given by the observation that expectations about rewards seem to induce a stronger effect than the reward magnitude.

In the present study, the fact of knowing about the reward disparities induces a clear modulatory effect. Our results also support previous studies suggesting that the effect of the monetary reward itself may enhance all the inhibitory system. Performances systematically improved after the starting block that was not monetarily rewarded. Improvement was seen from the first rewarded block, leading to a discrete progression of performances after that point when participants expected the reward shifts. Although, for the unexpected shift group, 
no other improvements were obtained after the first monetary reward given the lack of new expectancies about the forthcoming rewards.

The expected reward shift effect can be partially assimilated to the effect of the anticipation of reward. Previous studies have stated that the announcement of high rewards for future performances inside a consecutive set of tasks can boost performance during intermediate tasks involving interference control: The Simon task [26]. The anticipation of reward boosting mechanism could be perceived in distinct behavioural adjustments depending on the type of experiment even if they belong to the generic group of inhibition paradigms $[27,28,29]$. Our results do not replicate this observation although we are using another task. Instead, we have observed an immediate and steady effect of the highest reward at the beginning of the task, when previous information or previous assumptions are made about the size of expected rewards. Participants receiving low rewards at the beginning did not exhibit any immediate performance boost on low reward blocks when higher later rewards were expected. These results seem more in tune to the undermining effect [39]. Receiving a low reward when you expect a high one in some point, may have induced a demotivation, similar to the removal of an attractive reward. In the present work, we have used the stop signal task, having a simple instruction. A dynamic algorithm adjusts to each subject performance in order to avoid learning strategies. This is one more reason why we stick to the term "kick start effect" as a distinct concept than anticipation of rewards, because no extra boost performance was observed in intermediate blocks while waiting for the biggest bonus. We propose that these two concepts correspond to distinct neural mechanisms. The boosting effect of a strong reward at the beginning of a task, has not been explored in motor/cognitive inhibition tasks like ours.

The "kick start effect" that we describe here, could induce a more immediate up regulation of dopamine release in a phasic pattern, stirred by a quick and salient monetary cue [30]. And the anticipation of rewards would rely on more complex cognitive circuits demanding prefrontal lobe structures through goal-directed behaviour [37] or subcortical- ventral striatum circuits [31] mediated by a tonic dopamine release [32].

Apparently, when subjects are told in advance about the different reward sizes, this information may induce a stronger influence of the order of presentation of rewards. Hence, we can also claim that "expectation matters". In everyday life, this can be reflected on video 
games personal choice. Even if you don't receive real money or gifts, people look for the excitement of winning any kind of reward: coins, candies, some aliens or zombies to kill, and so on $[33,36]$. What matters here is the inner value you give to what you expect to get, according to intrinsic motivation.

Furthermore, our results are consistent with predictive coding theory [25,34,35]. In our experiments, analyses of variances showed steadier values on the first blocks, which accounts for only certain information of the task on both trials. After the first block, we can suppose that a screening system of outcomes is in place, waiting for the moment when the highest reward is going to appear. Moreover, on the unexpected reward shift group, a single reward is expected without any other particular belief. Participants are not aware of the presence of reward magnitude differences. Variances are also shorter in the first block, beyond that, they become high and unstable, except on decreasing conditions, when the high reward at the beginning can be perceived as "strong enough" (500\$ enticing Colombian pesos). All these observations are also consistent with the hypothesis proposed by Ide and colleagues about rational decision-making in inhibitory control [4], based on optimal prediction of outcomes that modulate inhibitory behaviour.

Uncertainty is an influential factor over behaviour modulation, although it is difficult to build up controlled experimental conditions to test it. Some authors have proposed computational models to assess adaptive behaviour [35].

The experimental design proposed here could be employed as an evaluation tool to assess behavioural adjustments in front of rewards under an uncertain environment. In order to better grasp the electrophysiological effects on reward modulation, we consider it more judicious to apply the expected reward shift experimental design on further studies on monetary reward effect of inhibition. On the other hand, unexpected motivational trials may give us an interesting research tool for the neural underpinnings of unconscious aspects of the motivational system.

\section{Compliance with Ethical Standards}

All procedures performed in the present study were in accordance with the ethical standards of the institutional and/or national research committee and with the 1964 Helsinki declaration and its later amendments or comparable ethical standards. An explicit information about the 
ethical procedure of the present work, is presented in the Method section, participants subsection.

Informed consent: An informed consent was obtained from all individual participants included in the study, as stated in the Methods section.

Funding: This research was supported by a Wellcome Trust Biomedical Research Fellowship WT093811MA

Authors contributions: P.M.H., M.S. and T.B. were responsible for the study design. M.C. conducted technical implementation of the task. P.M.H., C.L.C. and M.B. contributed to data acquisition. P.M.H. and T.B. performed the data analysis. P.M.H. drafted the manuscript., M.S, A.V.V.M. and T.B. assisted with the interpretation of the findings and revision of the manuscript. All authors critically reviewed the content and approved the final version for publication.

Conflict of Interest: The authors declare no conflict of interest of competing financial interests.

\section{Acknowledgements}

We thank Dr Leonardo Palacios and Dr Julio Ponce de León for facilitating data acquisition on their departments, Dr Oliver Müller for helpful technical and data acquisition support, $\operatorname{Pr}$ Srivas Chennu for helpful data analysis and manuscript discussion.

\section{References}


1. Aron, A. R., Fletcher, P. C., Bullmore, E. T., Sahakian, B. J., \& Robbins, T. W. (2003). Stop-signal inhibition disrupted by damage to right inferior frontal gyrus in humans. Nature neuroscience, 6(2), 115-116.

2. Dempster, F. N. (1992). The rise and fall of the inhibitory mechanism: Toward a unified theory of cognitive development and aging. Developmental review, 12(1), $45-$ 75.

3. Bari, A., \& Robbins, T. W. (2013). Inhibition and impulsivity: behavioral and neural basis of response control. Progress in neurobiology, 108, 44-79.

4. Ide, J. S., Shenoy, P., Angela, J. Y., \& Chiang-shan, R. L. (2013). Bayesian prediction and evaluation in the anterior cingulate cortex. The Journal of Neuroscience, 33(5), 2039-2047.

5. Alderson, R. M., Rapport, M. D., \& Kofler, M. J. (2007). Attentiondeficit/hyperactivity disorder and behavioral inhibition: a meta-analytic review of the stop-signal paradigm. Journal of abnormal child psychology, 35(5), 745-758.

6. van den Wildenberg, W. P., van der Molen, M. W., \& Logan, G. D. (2002). Reduced response readiness delays stop signal inhibition. Acta psychologica, 111(2), 155-169.

7. Nigg, J. T., \& Casey, B. J. (2005). An integrative theory of attentiondeficit/hyperactivity disorder based on the cognitive and affective neurosciences. Development and psychopathology, 17(03), 785-806.

8. MacLeod, C. M. (2007). The concept of inhibition in cognition. Inhibition in cognition, 3-23.

9. Anderson, V. (1998). Assessing executive functions in children: Biological, psychological, and developmental considerations. Neuropsychological rehabilitation, 8(3), 319-349..

10. Leotti, L. A., \& Wager, T. D. (2010). Motivational influences on response inhibition measures. Journal of Experimental Psychology: Human Perception and Performance, 36(2), 430. 
11. Pessiglione, M., Schmidt, L., Draganski, B., Kalisch, R., Lau, H., Dolan, R. J., \& Frith, C. D. (2007). How the brain translates money into force: a neuroimaging study of subliminal motivation. Science, 316(5826), 904-906.

12. Ridderinkhof, K. R., Van Den Wildenberg, W. P., Segalowitz, S. J., \& Carter, C. S. (2004). Neurocognitive mechanisms of cognitive control: the role of prefrontal cortex in action selection, response inhibition, performance monitoring, and reward-based learning. Brain and cognition, 56(2), 129-140.

13. Huang-Pollock, C. L., Mikami, A. Y., Pfiffner, L., \& McBurnett, K. (2007). ADHD subtype differences in motivational responsivity but not inhibitory control: evidence from a reward-based variation of the stop signal paradigm. Journal of Clinical Child and Adolescent Psychology, 36(2), 127-136.

14. Jin, X., \& Costa, R. M. (2010). Start/stop signals emerge in nigrostriatal circuits during sequence learning. Nature, 466(7305), 457-462.

15. Seo, H., \& Lee, D. (2007). Temporal filtering of reward signals in the dorsal anterior cingulate cortex during a mixed-strategy game. The Journal of Neuroscience, 27(31), 8366-8377.

16. Alexander, W. H., \& Brown, J. W. (2010). Competition between learned reward and error outcome predictions in anterior cingulate cortex. Neuroimage, 49(4), 32103218 .

17. Herrera, P. M., Speranza, M., Hampshire, A., \& Bekinschtein, T. A. (2014). Monetary rewards modulate inhibitory control. Frontiers in human neuroscience, 8.

18. Brown, J. W., \& Braver, T. S. (2005). Learned predictions of error likelihood in the anterior cingulate cortex. Science, 307(5712), 1118-1121.

19. Jessup, R. K., Busemeyer, J. R., \& Brown, J. W. (2010). Error effects in anterior cingulate cortex reverse when error likelihood is high. The Journal of Neuroscience, 30(9), 3467-3472.

20. Logan, G. D., \& Cowan, W. B. (1984). On the ability to inhibit thought and action: A theory of an act of control. Psychological review, 91(3), 295.

21. Shenoy, P., \& Angela, J. Y. (2011). Wherefore a Horse Race: Inhibitory Control as Rational Decision Making. Neural Basis of Motivational and Cognitive Control, 371.

22. Shenoy, P., \& Yu, A. J. (2011). Rational decision-making in inhibitory control. Frontiers in human neuroscience, 5, 48. 
23. Rubia, K., Smith, A. B., Brammer, M. J., \& Taylor, E. (2003). Right inferior prefrontal cortex mediates response inhibition while mesial prefrontal cortex is responsible for error detection. Neuroimage, 20(1), 351-358.

24. Congdon, E., Mumford, J. A., Cohen, J. R., Galvan, A., Canli, T., \& Poldrack, R. A. (2012). Measurement and reliability of response inhibition. Frontiers in psychology, 3, 37.

25. Hohwy, J. (2013). The predictive mind. Oxford University Press.

26. Zedelius, C. M., Veling, H., Bijleveld, E., \& Aarts, H. (2012). Promising high monetary rewards for future task performance increases intermediate task performance. PloS one, 7(8), e42547.

27. Miller, J. (1987). Evidence of preliminary response preparation from a divided attention task. Journal of Experimental Psychology: Human Perception and Performance, 13(3), 425.

28. Krebs, R., Hopf, J. M., \& Boehler, C. N. (2015). Within-trial effects of stimulusreward associations. Motivation and Cognitive Control, 65.

29. Liljeholm, M., \& O'Doherty, J. P. (2012). Anything you can do, you can do better: neural substrates of incentive-based performance enhancement. PLoS Biol, 10(2), e1001272.

30. Berns, G. S., McClure, S. M., Pagnoni, G., \& Montague, P. R. (2001). Predictability modulates human brain response to reward. The journal of neuroscience, 21(8), 27932798 .

31. Tamietto, M., \& De Gelder, B. (2010). Neural bases of the non-conscious perception of emotional signals. Nature Reviews Neuroscience, 11(10), 697-709.

32. Schultz, W. (2002). Getting formal with dopamine and reward. Neuron, 36(2), 241263.

33. de Gortari, A. B. O., \& Griffiths, M. D. (2014). Automatic mental processes, automatic actions and behaviours in Game Transfer Phenomena: An empirical selfreport study using online forum data. International journal of mental health and addiction, 12(4), 432-452.

34. Schultz, W., \& Dickinson, A. (2000). Neuronal coding of prediction errors. Annual review of neuroscience, 23(1), 473-500.

35. Mathys, C., Daunizeau, J., Friston, K. J., \& Stephan, K. E. (2011). A Bayesian foundation for individual learning under uncertainty. Frontiers in human neuroscience, 5, 39. 
36. Habgood, M. P. J., Ainsworth, S. E., \& Benford, S. (2005). Endogenous fantasy and learning in digital games. Simulation \& Gaming, 36(4), 483-498.

37. Staudinger, M. R., Erk, S., \& Walter, H. (2011). Dorsolateral prefrontal cortex modulates striatal reward encoding during reappraisal of reward anticipation. Cerebral cortex, 21(11), 2578-2588.

38. Reeve, J., \& Lee, W. (2012). Neuroscience and human motivation. The Oxford handbook of human motivation, 365-380.

39. Murayama, K., Matsumoto, M., Izuma, K., \& Matsumoto, K. (2010). Neural basis of the undermining effect of monetary reward on intrinsic motivation. Proceedings of the National Academy of Sciences, 107(49), 20911-20916.

40. Pessiglione M, Schmidt L, Draganski B, Kalisch R, Lau H, Dolan RJ, \& Frith CD. How the brain translates money into force: a neuroimaging study of subliminal motivation. Science. 2007;316:5826. 